\section{SAFETY AND EFFICACY OF VIDEO-ASSISTED THORACIC SURGICAL TECHNIQUES FOR THE TREATMENT OF SPONTANEOUS PNEUMOTHORAX}

Video-assisted thoracic surgery has been widely used in the treatment of spontaneous pneumothorax despite a paucity of data regarding the relative safety and long-term efficacy for this procedure. We reviewed 113 consecutive patients (68 male and 45 female patients, aged 15 to 92 years, mean 35.1) who underwent 121 video-assisted thoracic surgical procedures during 119 hospitalizations from 1991 through 1993. Recurrent ipsilateral pneumothorax was the most frequent indication for surgery and occurred in 77 patients $(65 \%)$. The most common method of management was stapling of an identified bleb in the lung, which was undertaken in $105(87 \%)$ patients. No operative deaths occurred. Complications included an air leak lasting longer than 5 days in $10(8 \%)$ patients, two of whom required second procedures for definitive management. No episodes of postoperative bleeding or empyema occurred. The postoperative stay ranged from 1 day to 39 days (median 3 days, average 4.3 days) and 99 patients $(84 \%)$ were discharged within 5 days. Mean follow-up was 13.1 months and ranged from 1 to 34 months. Eleven patients $(10 \%)$ were lost to follow-up. Ipsilateral pneumothorax recurred after five of 121 procedures $\mathbf{( 4 . 1 \% )}$ ). Twelve perioperative parameters (age, gender, race, smoking history, site of pneumothorax, severity of pneumothorax, operative indications, number of blebs, site of blebs, bleb ablation, method of pleurodesis, and prolonged postoperative air leak) were entered into univariate and multivariate analysis to identify significant independent predictors of recurrence. The only independent predictor of recurrence was the failure to identify and ablate a bleb at operation, which resulted in a $23 \%$ recurrence rate versus a $1.8 \%$ rate in those with ablated blebs $(p<0.001)$. These data suggest that video-assisted thoracic surgery is a viable alternative to thoracotomy for the treatment of recurrent spontaneous pneumothorax. It results in a short hospital stay, low morbidity, high patient acceptance, and a low rate of recurrence. (J THORAC CaRdiovasC SuRg 1995;109:1198-204)

Keith S. Naunheim, MD, ${ }^{a}$ Michael J. Mack, MD, ${ }^{b}$ Steven R. Hazelrigg, MD, Mark K. Ferguson, MD, ${ }^{\mathrm{d}}$ Peter F. Ferson, MD, ${ }^{\mathrm{e}}$ Theresa M. Boley, RN, ${ }^{\mathrm{c}}$ and Rodney J. Landreneau, MD, ${ }^{f}$ St. Louis, Mo., Dallas, Tex., Springfield and Chicago, Ill., and Pittsburgh, $\mathrm{Pa}$.
$T^{1}$ he recent rediscovery of thoracoscopy involving advanced video technology has led to widespread application of this technique for a variety of thoracic clinical problems. One of the earliest uses was the application of video-assisted thoracic surgical

From the Departments of Surgery of St. Louis University Health Sciences Center, St. Louis, Mo., ${ }^{\text {a }}$ Medical City Hospital, Dallas, Tex., Southern Illinois University, Springfield, Ill., University of Chicago, Chicago, Ill., ${ }^{\mathrm{d}}$ and University of Pittsburgh, Pittsburgh, $\mathrm{Pa}$.

Read at the Twentieth Annual Meeting of The Western Thoracic Surgical Association, Olympic Valley, Calif., June 22-25, 1994.

Address for reprints: Keith S. Naunheim, MD, Professor of Surgery, St. Louis University Health Sciences Center, 3635 Vista Ave. at Grand Blvd., St. Louis, MO 63110-0250.

Copyright (C) 1995 by Mosby-Year Book, Inc.

$0022-5223 / 95 \$ 3.00+0 \quad \mathbf{1 2 / 6 / 6 2 9 4 6}$
(VATS) techniques for the treatment of patients with spontaneous pneumothorax. Although it has been suggested that VATS is ideally suited for the treatment of this problem and should be the procedure of choice, ${ }^{1,2}$ no report has been published to document the efficacy of the procedure (e.g., freedom from recurrent pneumothorax) in a large patient cohort over a prolonged follow-up period. This study was undertaken to assess the safety and efficacy of a VATS approach for pneumothorax.

\section{Patients and methods}

All patients undergoing a VATS procedure for the treatment of spontaneous pneumothorax were identified through a review of records at St. Louis University Health Sciences Center, Medical City Hospital in Dallas, the University of Chicago Hospitals, Southern Illinois University Medical Center at Springfield, Illinois, and the Uni- 
versity of Pittsburgh Medical Center. Office charts and hospital records were reviewed to identify pertinent demographic data including age, race, and gender. Clinical data recorded included smoking history and duration (as recorded in pack-years), the laterality of pneumothorax (left, right, or bilateral), and the extent of pneumothorax (up to $25 \%, 26 \%$ to $50 \%, 51 \%$ to $75 \%, 76 \%$ to $100 \%$, or tension pneumothorax) on the initial chest $\mathrm{x}$-ray film. Operative data noted included indication for operation (uncomplicated first episode, recurrent ipsilateral pneumothorax, recurrent contralateral pneumothorax, persistent air leak, hemothorax, or tension pneumothorax), the number of blebs identified (none, one, or multiple), and the site of blebs (upper lobe, middle lobe, lower lobe, or multiple sites). The procedure performed was divided into the method of management of the pulmonary blebs and the method of pleurodesis if performed. Bleb management included identification and stapling of blebs, identification and ablation of blebs by other methods (laser, ligature, or cautery), blind stapling of the apex when no bleb was identified, or pleurodesis only with no lung manipulation. The pleural procedures coded for included gauze abrasion, instillation of talc or other sclerotic chemicals, laser pleurodesis, partial pleurectomy, or no procedure. Operative complications and length of stay were also abstracted from the hospital charts.

Follow-up was conducted during clinic visits or telephone conversation with patients, their families, or their primary-care physicians. Inquiries were made regarding recurrence of pneumothorax and new pulmonary symptoms.

Statistical analysis was undertaken to identify predictors of recurrent pneumothorax. An initial univariate analysis was performed with the use of Student's $t$ test for continuous variables and a $2 \times \mathrm{k}$ contingency table for categoric variables. Parameters that were significant or marginally significant $(p<0.10)$ as predictors for recurrence were then entered into a stepwise linear regression analysis to identify independent predictors of recurrent pneumothorax. Actuarial freedom from recurrence was analyzed by means of a Berkson-Gage life-table model.

\section{Results}

One hundred thirteen consecutive patients were identified who had undergone a VATS procedure for the treatment of spontaneous pneumothorax at the five participating institutions between February 1991 and December 1993. The group comprised 68 male and 45 female patients whose ages ranged from 15 to 92 years with a mean of 35.1 years. Ninety-five patients were white $(84 \%), 15$ were black $(13 \%)$, and three $(3 \%)$ were Hispanic or Oriental. Forty-seven patients $(39.5 \%)$ had a history of smoking, with a mean of 12.6 pack-years among those who smoked. During the 119 hospitalizations, there were 52 left pneumothoraces (44\%), 65 right $(55 \%)$, and two bilateral pneumothoraces (2\%). In 11 cases the extent of pneumothorax could not be
Table I. Operative indications, findings, and management

\begin{tabular}{lrr}
\hline & No. & $\%$ \\
\hline Surgical indications & & \\
Ipsilateral recurrence & 77 & 65 \\
Persistent air leak & 24 & 20 \\
Contralateral recurrence & 12 & 10 \\
Uncomplicated first episode & 3 & 3 \\
Hemothorax & 2 & 2 \\
Number of blebs & & \\
None & 11 & 9 \\
One & 35 & 29 \\
Multiple & 75 & 62 \\
Site of blebs & & \\
Upper lobe & 88 & 73 \\
Middle lobe & 2 & 2 \\
Lower lobe & 9 & 7 \\
Multiple sites & 11 & 9 \\
Not stated & 11 & 9 \\
Bleb management & & \\
Bleb stapling & 105 & 87 \\
Blind apical stapling & 8 & 7 \\
Pleurodesis only & 5 & 4 \\
Other bleb ablation & 3 & 3 \\
$\quad$ (laser 1, ligature 1, cautery 1) & & \\
Pleural procedure & & \\
Gauze abrasion & 54 & 45 \\
Chemical pleurodesis & 19 & 16 \\
Laser pleurodesis & 16 & 13 \\
None & 12 & 10 \\
Pleurectomy & 10 & 8 \\
Talc pleurodesis & 8 \\
\hline & & \\
\hline
\end{tabular}

determined from review of the records. The size of the pneumothorax in the remaining episodes was up to $25 \%$ in $40(37 \%), 26 \%$ to $50 \%$ in $35(32 \%), 51 \%$ to $75 \%$ in $13(12 \%), 76 \%$ to $100 \%$ in $17(16 \%)$, and tension pneumothorax was present in three instances (3\%).

The surgical indications, as well as the operative findings and management, are detailed in Table I. The most frequent indication for operative intervention was an ipsilateral recurrent pneumothorax, which was noted in 77 patients $(65 \%)$. Four patients (3\%) underwent VATS procedures for an uncomplicated first episode of spontaneous pneumothorax. In the majority of patients $(62 \%)$ more than one bleb was visualized. However, in 11 patients $(9 \%)$ no bleb or bulla could be identified. The most common site for blebs in the 110 procedures in which they were identified was the upper lobe $(80 \%)$. Blebs were found in more than one lobe during 11 procedures $(10 \%)$.

Management of the lung in the 121 procedures performed included stapling (with or without exci- 


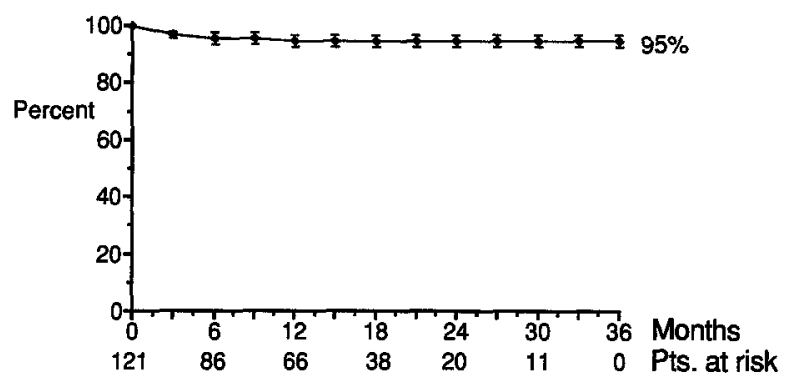

Fig. 1. Actuarial freedom from recurrent pneumothorax after VATS procedure. Error bars indicate plus or minus one standard error.

sion) of an identified bleb in 105 patients (87\%). Three additional patients had blebs that were ablated by laser ablation, cautery, and ligature in one patient each. The final two patients in whom blebs were visualized underwent gauze abrasion pleurodesis only. Management in the 11 patients with no identifiable bleb included pleurodesis by gauze abrasion in eight patients, chemical instillation in two, and apical pleurectomy in one. Eight patients also underwent a "blind" stapling of the apex.

Pleural procedures performed included gauze abrasion in 54 (45\%), instillation of sclerotic chemicals (tetracycline, doxycycline) in $19(16 \%)$, talc instillation in $10(8 \%)$, laser sclerosis in $16(13.2 \%)$, and partial pleurectomy in $10(8 \%)$. In 12 procedures $(10 \%)$ no attempt at pleurodesis was undertaken.

No operative deaths occurred and no procedure was converted to a thoracotomy at the initial setting. Ten patients $(8 \%)$ had an air leak lasting longer than 5 days. Two of these patients underwent second operative procedures (one VATS, one thoracotomy) for definitive management of the air leaks. In no case did bleeding, empyema, ventilator dependence, stroke, or myocardial infarction occur. The postoperative stay ranged from 1 to 39 days with a median of 3 days and a mean of $4.3 \pm 4.5$ days. Ninety-nine patients $(84 \%)$ were discharged within 5 days after the procedure.

Patients were followed up between 1 and 34 months with a mean follow-up of 13.1 months. Eleven patients $(10 \%)$ were lost to follow-up. Recurrent ipsilateral pneumothorax occurred after five of the 121 VATS procedures $(4.1 \%)$. These occurred at 2, 4, 7, 13, and 52 weeks after the original VATS procedure. Actuarial freedom from recurrence is depicted in Fig. 1. The recurrent pneumo- thoraces were managed in a variety of fashions. In one patient with a small $(10 \%)$ loculated pneumothorax, observation only was undertaken. One patient was treated only with a chest tube, two underwent repeat VATS procedures with pleurodesis, and the final patient underwent thoracotomy with partial pleurectomy.

Twelve perioperative parameters were entered into univariate analysis to identify univariate predictors of recurrence. These included age, gender, race, smoking history, site of pneumothorax, severity of pneumothorax, operative indications, number of blebs, site of blebs, bleb ablation, method of pleurodesis, and the presence of a prolonged postoperative air leak. Only two parameters proved to be univariate predictors of recurrence: the number of identified blebs and ablation or nonablation of blebs. The recurrence rate after procedures in which multiple blebs were identified was $2.7 \%$ (2/75). When only one bleb was visualized it was $0 \%(0 / 35)$ and when no blebs were identified the incidence of recurrence was $27.3 \%(3 / 11)(p=0.0002)$. In procedures during which identified blebs were ablated there was a $1.8 \%(2 / 108)$ incidence of recurrence versus a $23 \%(3 / 13)$ incidence for those in which no bleb was identified and ablated $(p=0.003)$. Stepwise linear regression revealed that the performance of bleb ablation was a significant independent predictor $(p<0.001)$.

\section{Discussion}

VATS has been received with an initial wave of enthusiasm that has led to the broad application of this technology to many thoracic arenas including diseases of the pericardium, pleura, lung, esophagus, thymus, and chest wall. ${ }^{3}$ Now that several years of experience have been accumulated with this technique, it appears time to assess the early results for the routine use of VATS for thoracic surgical problems.

Historically, operative intervention for recurrent spontaneous pneumothorax has been undertaken via standard posterolateral thoracotomy, anterior thoracotomy, sternotomy, and axillary minithoracotomy. This last approach has become very popular and, before VATS, was probably the most commonly used approach. Regardless of the approach, the operation performed was identification and ablation of pulmonary blebs via stapling or suture ligation.

The point of greatest controversy arose over the need for and method of promoting adequate pleural 
symphysis. Methods of pleurodesis have included mechanical abrasion with gauze, the instillation of chemical irritants (tetracycline, doxycycline, bleomycin), pleural irritation with a laser or cautery, and the instillation of talc. Recent experimental work by Bresticker and colleagues ${ }^{4}$ compared the pleural adhesions promoted by the aforementioned methods. Talc instillation and gauze abrasion proved effective and superior to laser, cautery, and tetracycline with regard to the quality and quantity of adhesions. Although equally effective, talc has been used less frequently than gauze abrasion because of fears of asbestos contamination and because of the excessive granulomatous inflammation it incites, which may make subsequent thoracotomy very difficulty. Mechanical pleural abrasion with a sterile gauze sponge (or similar rough surface) has been long used and has a high rate of success as evidenced by a $0 \%$ to $5 \%$ recurrence rate..$^{5-9}$ This is currently our method of choice for pleurodesis with the VATS approach.

Total pleurectomy is also effective for pleurodesis but has some disadvantages that have been addressed by "downsizing" the procedure to partial or apical pleurectomy. ${ }^{10-12} \mathrm{~A}$ report by Deslauriers and colleagues ${ }^{13}$ presented the results of over 400 transaxillary procedures in which apical pleurectomy was performed after ablation of pulmonary blebs. The rate of ipsilateral pneumothorax recurrence was slightly less than $1 \%$, but three patients required reoperation for bleeding. This complication of pleurectomy has been reported by others and is one of the most frequent criticisms of the procedure. ${ }^{12,14,15}$ One additional criticism of pleurectomy is that it will make a subsequent thoracotomy exceedingly difficult should any of these young patients require thoracic surgical intervention in future years. ${ }^{10,11}$

Although the application of VATS for the treatment of pneumothorax has been relatively recent, rigid thoracoscopy has been used in the assessment and management of this disorder for three decades. ${ }^{16,17}$ The evolution from rigid thoracoscopy to VATS has occurred within the past 5 years. ${ }^{18}$ During this time the methods used for bleb ablation have also evolved from lasers ${ }^{19}$ to cautery ${ }^{20}$ or ligature ${ }^{21}$ and, finally, to mechanical stapling. ${ }^{22}$ With the introduction of endoscopic staplers, proponents of the VATS approach to spontaneous pneumothorax can assert that they are performing the same operation that they had used previously and have changed only the approach.

Our experience documents that a VATS ap- proach is safe. The major complication encountered was an $8 \%$ incidence of prolonged air leak. This required a second procedure in two instances $(1.7 \%)$, a reoperation rate not dissimilar to those in the reports of Brooks ${ }^{6}$ (3.3\%), Granke ${ }^{9}(1.3 \%)$, Deslauriers ${ }^{13}(1.0 \%)$, and their associates. Although our mean postoperative stay of 4.3 days is shorter than that reported for either a standard thoracotomy or transaxillary approach, ${ }^{4-9}$ the results may not be directly comparable because of differing patient populations and changes in discharge policies that have occurred over the past several years as a result of external pressures. However, even when compared with a very recent report of 14 highly selected patients (less than 30 years of age, no concomitant medical problems) treated via a transaxillary approach, our mean postoperative stay compares favorably (4.3 versus 5.1 days).

Our recurrence rate of $4.1 \%$ falls within the accepted recurrence rate of patients treated for recurrent pneumothorax via an open approach. It is also similar to the incidence reported by Yamaguchi and associates ${ }^{23}(2.7 \%)$ and Inderbitzi and Furrer ${ }^{24}$ $(4.5 \%)$ in their early reports using VATS techniques. Our risk analysis did, however, reveal a worrisomely high recurrence rate in those patient in whom no bleb could be identified and ablated. It is in such patients that apical pleurectomy may be indicated. This could be performed at the same setting either via a VATS approach or via an axillary thoracotomy and would perhaps provide a more definitive pleural symphysis with a decreased likelihood of subsequent recurrence. We believe this approach should be strongly considered when no pulmonary disease can be visualized for the purpose of ablation.

Unfortunately, spontaneous pneumothorax is a common problem and as such accounts for a considerable expenditure of money and resources. ${ }^{25}$ Much of the enthusiasm for VATS has come from the notion that minimally invasive surgery will shorten overall hospital stay, as well as lower total hospital cost and resource expenditure. We did not specifically examine charges or costs in these patients. This would have been exceedingly difficult given involvement of six institutions in different areas of the county. However, prior analyses of minimally invasive surgery both within the abdo$m^{26}{ }^{26}$ and pertaining to open lung biopsy in the chest $^{27}$ have been undertaken in an attempt to identify relative charges and costs. Although hospital stay is generally shorter with the minimally 
invasive technique, the increased expenditure associated with the technology (reusable and disposable equipment) and prolonged operative times have resulted in no net savings with regard to hospital charges. However, a full analysis of the cost-benefit relationship of VATS treatment of pneumothorax will need to take into account hospital charges, lost employment hours, and quality of life to provide valid answers regarding the appropriateness of a VATS approach.

Our experience suggests that VATS is a viable alternative for the treatment of recurrent spontaneous pneumothorax. We believe it is the treatment of choice when the decision is made for surgical intervention. The procedure is well tolerated and allows for early discharge in 3 days or less in more than half the patients. The $4.1 \%$ recurrence rate is consistent with rates of recurrence found when an open thoracotomy technique with gauze abrasion is used. As experience grows with the VATS technique, one might see a decrease in the rate of recurrence as a result of a plateau in the learning curve. However, the current rate of recurrence appears acceptable, and we believe that VATS is an excellent initial approach for this common problem.

\section{REFERENCES}

1. Melvin WS, Krasna MJ, McLaughlin JS. Thoracoscopic management of spontaneous pneumothorax. Chest 1992;102:1877-9.

2. Takeno Y. Thoracoscopic treatment of spontaneous pneumothorax. Ann Thorac Surg 1993;56:688-90.

3. Hazelrigg SR, Nunchuck SK, LoCicero J. Video Assisted Thoracic Surgery Study Group Data. Ann Thorac Surg 1993;56:1039-43.

4. Bresticker MA, Oba J, LoCicero J, Greene R. Optimal pleurodesis: a comparison study. Ann Thorac Surg 1993;55:364-7.

5. Youmans CR, Williams RD, McMinn MR, Derrick LR. Surgical management of spontaneous pneumothorax by bleb ligation and pleural dry sponge abrasion. Am J Surg 1970;120:644-8.

6. Brooks JW. Open thoracotomy in the management of spontaneous pneumothorax. Ann Surg 1973;177:798805.

7. Murray KD, Mathey RG, Howanitz EP, Myerowitz PD. A limited axillary thoracotomy as primary treatment for recurrent spontaneous pneumothorax. Chest 1993;103:137-42.

8. Thomas P, LeMee F, LeHors H, et al. Results of surgical treatment of persistent or recurrent pneumothorax. Ann Chir 1993;47:136-40.

9. Granke K, Fischer CR, Gago O, Morris JD, Prager
RL. The efficacy and timing of operative intervention for spontaneous pneumothorax. Ann Thorac Surg 1986;42:540-2.

10. Gaensler EA. Partial pleurectomy for recurrent spontaneous pneumothorax. Surg Gynecol Obstet 1956; 102:293-7.

11. Singh SU. Current status of parietal pleurectomy in recurrent pneumothorax. Scand J Thorac Cardiovasc Surg 1979;13:93-9.

12. Weeden D, Smith GH. Surgical experience in the management of spontaneous pneumothorax 1972-82. Thorax 1983;38:737-40.

13. Deslauriers J, Beaulieu M, Despres JP, et al. Transaxillary pleurectomy for treatment of spontaneous pneumothorax. Ann Thorac Surg 1980;30:569-75.

14. Feld RS, Levin DC, Grey DP, Mee RB. Transcatheter embolization for control of bleeding following pleurectomy. Ann Thorac Surg 1979;27:363-6.

15. Thomas PA, Gebauer PW. Results and complications of pleurectomy for bullous emphysema and recurrent pneumothorax. J Thorac Cardiovasc Surg 1960;39: 194-9.

16. Van de Brekel JA, Duurkens VA, Vanderschueren RG. Pneumothorax: results of thoracoscopy and pleurodesis with the poudrage and thoracotomy. Chest 1993;103:345-7.

17. Tsukamoto $\mathrm{T}$, Nakamura $\mathbf{H}$, Satoh $\mathrm{T}$, Yamada $\mathrm{K}$, Nagasawa M. Comparative studies using a rigid thoracoscope and fiberoptic bronchoscope to treat spontaneous pneumothorax. Chest 1991;100:953-8.

18. Daniel TM, Tribble CG, Rodgers BM. Thoracoscopy and talc poudrage for pneumothoraces and effusions. Ann Thorac Surg 1990;50:186-9.

19. Torre $\mathrm{M}$, Belloni P. Nd:YAG laser pleurodesis through thoracoscopy: new curative therapy in spontaneous pneumothorax. Ann Thorac Surg 1989;47:887-9.

20. Wakabayashi A. Thoracoscopic ablation of blebs in the treatment of recurrent or persistent spontaneous pneumothorax. Ann Thorac Surg 1989;48:651-3.

21. Nathanson LK, Shimi SM, Wood RA, Cuschieri A. Videothoracoscopic ligation of bulla and pleurectomy for spontaneous pneumothorax. Ann Thorac Surg 1991;52:316-9.

22. Cannon WB, Vierra MA, Cannon A. Thoracoscopy for spontaneous pneumothorax. Ann Thorac Surg 1993;56:686-7.

23. Yamaguchi A, Shinonaga M, Tatebe S, et al. Thoracoscopic stapled bullectomy supported by suturing. Ann Thorac Surg 1993;56:691-3.

24. Inderbitzi R, Furrer $M$. The surgical treatment of spontaneous pneumothorax by video-thoracoscopy. Thorac Cardiovasc Surg 1992;40:330-3.

25. Bense L, Wiman LG, Jendteg S, Lindgren B. Economiccosts of spontaneous pneumothorax. Chest 1991;99:260-1.

26. Stoker ME, Vose J, O’Mara P, Maini BS. Laparo- 
scopic cholecystectomy: a clinical and financial analysis of 280 operations. Arch Surg 1992;127:589-94.

27. Hazelrigg SR, Nunchuck SK, Mack MJ, et al. Cost analysis for thoracoscopy: thoracoscopic wedge resection. Ann Thorac Surg 1993;56:633-5.

\section{Discussion}

Dr. Walter B. Cannon (Palo Alto, Calif.). VATS has now matured and become part of the daily armamentarium of thoracic surgeons throughout this country. Therefore, we have now reached the time to reflect and review its usefulness as a procedure in these times of costly medical care. As laparoscopy became the ideal therapeutic procedure for cholecystectomy, VATS has become the ideal procedure for bleb resection for spontaneous pneumothorax. With the advent of linear stapling devices and minicameras, the technical procedure became easy for the physician to perform and easy for the patient to tolerate. These authors have tried to prove that it is indeed the procedure of choice for patients who have spontaneous pneumothorax, and in my opinion they have done so.

We at Stanford have tried over the past few years to devise ways of reducing the hospitalization time for these patients, and we believe that two adjuncts can be added to the procedure to reduce the hospital stay. The first is installation of $0.5 \%$ bupivacaine (Marcaine) into the chest tube at the completion of the procedure. This seems to reduce the pain in these patients in the early postoperative period. We have also begun to use much smaller chest tubes, usually No. 20 , that we remove within 24 hours if there is no air leak and the lung is fully expanded. By using this method, we are able to discharge most patients with spontaneous pneumothorax the next day.

We would agree with the authors about pleurodesis. We have not been happy with the argon beam coagulator or laser or even cautery and have resumed using either a sponge on a ring forceps or a small tonsil sponge on a holder. We do not like using talcum powder in young healthy patients because of the risk of long-term harmful effects. Limited pleurectomy has increased risks but may be necessary in some patients, as pointed out by the authors. For some reason we seem to see a large number of patients with cystic fibrosis who have a spontaneous pneumothorax. Resection of these blebs in these patients has yielded surprisingly good results. Because of the potential for lung transplantation we have not done any pleural procedures at all because the risk of recurrent spontaneous pneumothorax may be higher. However, our experience has not borne out that concern. These patients so far have had durable results. None of these patients has had lung transplantation, so we do not know how problematic the pleural adhesions will be.

Aggressive treatment of spontaneous pneumothorax at the first event has occasionally been suggested, as in three patients whom Dr. Naunheim mentioned. We believe that $75 \%$ of patients who have a spontaneous pneumothorax will never have another one. For those patients, therefore, we do not believe a bleb resection at the first event is appropriate. However, we agree with the authors that spontaneous pneumothorax and persistent air leak or bleeding are definite indications for the procedure. Al- though the cost of the procedure may be higher, if we assess the total cost to society, as the cardiac surgeons have done, these patients become productive citizens in less time. They are hospitalized for shorter periods and therefore the drain on other medical resources is less. I believe the procedure is the best one that we have to offer these patients in the setting of health care costs in this country.

I would like to ask a couple of questions of Dr. Naunheim. Have you used ventilation scans to determine the location of an air leak, particularly in a patient who has a persistent air leak and may need another VATS or open procedure? Have you any tricks that you can use to find the air leak during a VATS procedure, which is difficult to do in comparison with doing an open procedure.

Dr. Naunheim. We have not used ventilation scans, although that is an ingenious idea. In the majority of cases we depend on visual identification of the leaking bleb. The only trick we use is one well known to everybody: we direct our attention to the apex and the superior segment of the lower lobe. The vast majority of leaks will be there. We put the patient in the Trendelenburg position, instill 50 to $75 \mathrm{ml}$ of saline solution, and have the anesthesiologist insufflate the lung. The problem is that the operative view becomes somewhat obstructed at that point. To alleviate this visual obstruction, we visualize the lung lobe by lobe. We insert the scope through the midaxillary trocar site, and through a posterior or an anterior site we insert sponge sticks and try to compress the middle and lower lobes during insufflation and keep them compressed. Then once again we visualize the middle lobe, trying to keep the upper lobe and lower lobe compressed. Through a combination of compression of the lung and positive insufflation of the lung, we attempt to find a leak just by following the bubbles. It does not always work. Sometimes we can be fooled if we have to compress the lobe we are interested in such that it becomes atelectatic and the leak is missed.

Dr. Marvin Pomerantz (Denver, Colo.). We have had six or seven patients with spontaneous pneumothoraces without apparent blebs. We have always resected the apex of the lung, and uniformly the pathologist finds cysts. In these cases, we use Marlex mesh (Bard Implants, Billerica, Mass.) on a ring forceps to get a better abrasion. We have been using this method for only $1 \frac{1}{2}$ years, but we have not noted any recurrences. Do you have any experience that is similar?

Dr. Naunheim. I must admit to being gratified when I find a bleb; however, when I cannot visualize such a lesion, I direct my therapy to the apex of the lung. I believe the odds are better than 50:50 that the bleb is in the apex even if I cannot find it. Thus, I staple the area and use gauze on a ring forceps to abrade the pleura. It may be that Marlex mesh is more abrasive and thus better. I cannot assert that one is better than the other, but I do believe blind apical stapling makes some sense. Very little parenchyma is lost, and more than $50 \%$ of the time the bleb will be located in the apex whether it is identified or not.

Dr. Robbin Cohen (Los Angeles, Calif.). I agree with Dr. Cannon that patients with uncomplicated first-time pneumothorax do not require surgical therapy. Would you 
elaborate about the patients on whom you operated for primary uncomplicated first-time pneumothorax?

From the age spread of your patients, I presume that some of them had primary spontaneous pneumothorax and that some of the older patients probably had secondary pneumothoraces as a result of chronic obstructive pulmonary disease. In our experience, older patients with chronic obstructive pulmonary disease are sicker and our results are not quite as good. What approach do you use in those patients? Are your results in patients with secondary pneumothorax similar to those obtained in younger patients with primary spontaneous pneumothorax?

Dr. Naunheim. The results have been essentially equal except that the patients who are older, with chronic obstructive pulmonary disease, tend to have a more prolonged hospitalization. The incidence of air leak, which is the complication that generally prolongs the hospitalization time, was the same for patients older and younger than 40 years. However, it takes longer to get older patients up and moving. Patients who are in their 70 s and 80 s cannot go home at 3 or 4 days.

The patients operated on for first-time uncomplicated pneumothorax were operated on early in the series. There was a great wave of enthusiasm with the advent of thoracoscopy, with suppositions being made that the scalpel would become a relic of the past and thoracotomy would be of historical interest only. It was on the basis of this zeal and enthusiasm that we undertook a VATS procedure on three patients with primary pneumothorax who did not fit the classic indications. I completely agree, as do all my coauthors, that patients with primary uncomplicated first-time pneumothorax have only a $20 \%$ or $25 \%$ chance of ever requiring any intervention. Such patients have a $75 \%$ chance of living their life comfortably without three small incisions on their chest, and there is no reason for us to provide those for them.

Dr. Paul Waters (Los Angeles, Calif.). I am not convinced that this is the procedure of choice for such patients. A 4- or 5-day mean hospital stay is the norm after regular transapical or transaxillary thoracotomy for the young patient with apical blebs. Do you have information on where the cost savings are?

Dr. Naunheim. In the manuscript we explain why we did not try to do a cost analysis. The study encompasses six different institutions spread over a 3-year period with different billing systems at different hospitals. Also, no one really has any idea about actual cost; all we can gather information on is charges.

The cost studies for thoracoscopy that I am aware of show no cost savings with regard to hospital charges. If VATS is going to offer an advantage, it is going to be with regard to patient pain, which we believe to be less with a VATS procedure. I do think the patients are going to be able to be discharged more quickly. We had patients as old as 92 years of age, and at least a quarter of our patients were older than 50 years of age. Many of these patients would have difficulty tolerating even a small thoracotomy. The vast majority of our patients under the age of 30 years are out of the hospital by 3 days. I have difficulty discharging a patient in 1 or 2 days after an axillary thoracotomy or any other kind of thoracotomy. I do believe we will eventually find that these patients return to work faster. I do not think VATS is going to be any cheaper, but I do think the patients will have less discomfort and perhaps will lose less time from work. I have no data to substantiate these assumptions, however. 